PROCEEDINGS OF THE

AMERICAN MATHEMATICAL SOCIETY

Volume 140, Number 1, January 2012, Pages 129-134

S 0002-9939(2011)10899-2

Article electronically published on May 20, 2011

\title{
CULLEN NUMBERS WITH THE LEHMER PROPERTY
}

\author{
JOSÉ MARÍA GRAU RIBAS AND FLORIAN LUCA \\ (Communicated by Matthew A. Papanikolas)
}

\begin{abstract}
Here, we show that there is no positive integer $n$ such that the $n$th Cullen number $C_{n}=n 2^{n}+1$ has the property that it is composite but $\phi\left(C_{n}\right) \mid C_{n}-1$.
\end{abstract}

\section{INTRODUCTION}

A Cullen number is a number of the form $C_{n}=n 2^{n}+1$ for some $n \geq 1$. They attracted the attention of researchers since it seems that it is hard to find primes of this form. Indeed, Hooley 8 showed that for most $n$ the number $C_{n}$ is composite. For more about testing $C_{n}$ for primality, see [3] and [6. For an integer $a>1$, a pseudoprime to base $a$ is a composite positive integer $m$ such that $a^{m} \equiv a$ $(\bmod m)$. Pseudoprime Cullen numbers have also been studied. For example, in [12, it is shown that for most $n, C_{n}$ is not a base $a$ pseudoprime. Some computer searches up to several millions did not turn up any pseudoprime $C_{n}$ to any base. Thus, it would seem that Cullen numbers which are pseudoprimes are very scarce. A Carmichael number is a positive integer $m$ which is a base a pseudoprime for any $a$. A composite integer $m$ is called a Lehmer number if $\phi(m) \mid m-1$, where $\phi(m)$ is the Euler function of $m$. Lehmer numbers are Carmichael numbers, hence, pseudoprimes in every base. No Lehmer number is known, although it is known that there are no Lehmer numbers in certain sequences, such as the Fibonacci sequence (see [9]), or the sequence of repunits in base $g$ for any $g \in[2,1000]$ (see [4]). For other results on Lehmer numbers, see 1, 2, [11, [13, [14.

Our result here is that there is no Cullen number with the Lehmer property. Hence, if $\phi\left(C_{n}\right) \mid C_{n}-1$, then $C_{n}$ is prime.

Theorem 1. Let $C_{n}$ be the nth Cullen number. If $\phi\left(C_{n}\right) \mid C_{n}-1$, then $C_{n}$ is prime.

\section{Proof of Theorem 1}

Assume that $n \geq 30$, that $\phi\left(C_{n}\right) \mid C_{n}-1$, but that $C_{n}$ is not prime. Then $C_{n}$ is square-free. Write

$$
C_{n}=\prod_{i=1}^{k} p_{i} .
$$

Received by the editors October 14, 2010 and, in revised form, November 11, 2010.

2010 Mathematics Subject Classification. Primary 11A05; Secondary 11N25, 11A07.

(C)2011 American Mathematical Society 
So,

$$
\prod_{i=1}^{k}\left(p_{i}-1\right) \mid n 2^{n} .
$$

Write $n=2^{\alpha} n_{1}$, where $n_{1}$ is odd. Then $C_{n}=n_{1} 2^{n_{2}}+1$, where $n_{2}:=\alpha+n$. Let $p$ be any prime factor of $C_{n}$. Since $p-1 \mid C_{n}-1$, it follows that $p=m_{p} 2^{n_{p}}+1$ for some odd divisor $m_{p}$ of $n$ and some $n_{p}$ with

$$
n_{p} \leq n_{2}=n+\alpha \leq n+\frac{\log n}{\log 2} .
$$

Let us first show that in fact $n_{p} \leq n$. Assume that $n_{p}>n$. Then,

$$
C_{n}=n 2^{n}+1=p \lambda,
$$

for some positive integer $\lambda$, where $p \geq 2^{n+1}+1$. Observe that $\lambda>1$ because $C_{n}$ is not prime. Now

$$
\lambda=\frac{C_{n}}{p} \leq \frac{n 2^{n}+1}{2^{n+1}+1}<n .
$$

Reducing equation (11) modulo $2^{n}$, we get that $2^{n} \mid \lambda-1$, so $2^{n} \leq \lambda-1<n$, which is false for any $n>1$. Hence, $n_{p} \leq n$.

Next we look at $m_{p}$. If $m_{p}=1$, then $p=2^{n_{p}}+1$ is a Fermat prime. Hence, $n_{p}=2^{\gamma_{p}}$ for some nonnegative integer $\gamma$. Since $2^{\gamma_{p}}=n_{p} \leq n$, we get that $\gamma_{p}<$ $(\log n) /(\log 2)$. Hence, the prime $p$ can take at most $1+(\log n) /(\log 2)$ values. Next, observe that since

$$
\prod_{p \mid C_{n}} m_{p} \mid n,
$$

it follows that the number of prime factors $p$ of $C_{n}$ such that $m_{p}>1$ is $\leq$ $(\log n) /(\log 3)$. Hence, we have arrived at the bound

$$
k<1+\frac{\log n}{\log 2}+\frac{\log n}{\log 3}<1+2.4 \log n .
$$

We next bound $n_{p}$. Put $N:=\lfloor\sqrt{n / \log n}\rfloor$, and consider pairs $(a, b)$ of integers in $\{0,1, \ldots, N\}$. There are $(N+1)^{2}>n / \log n$ such pairs. For each such pair, consider the expression $L(a, b):=a n+b n_{p} \in\left[0,2 n^{3 / 2} /(\log n)^{1 / 2}\right]$. Thus, there exist two pairs $(a, b) \neq\left(a_{1}, b_{1}\right)$ such that

$$
\left|\left(a-a_{1}\right) n+\left(b-b_{1}\right) n_{p}\right|=\left|L(a, b)-L\left(a_{1}, b_{1}\right)\right| \leq \frac{2 n^{3 / 2} /(\log n)^{1 / 2}}{n / \log n-1}<3(n \log n)^{1 / 2} .
$$

Put $u:=a-a_{1}, v:=b-b_{1}$. Then $(u, v) \neq(0,0)$ and

$$
\left|u n+v n_{p}\right|<3(n \log n)^{1 / 2} .
$$

We may also assume that $u$ and $v$ are coprime, for if not, we replace the pair $(u, v)$ by the pair $\left(u_{1}, v_{1}\right)$, where $d:=\operatorname{gcd}(u, v), u_{1}:=u / d, v_{1}:=v / d$, and the properties that $\max \left\{\left|u_{1}\right|,\left|v_{1}\right|\right\} \leq(n / \log n)^{1 / 2}$ and $\left|u_{1} n+v_{1} n_{p}\right|<3(n \log n)^{1 / 2}$ are still fulfilled. Finally, up to replacing the pair $(u, v)$ by the pair $(-u,-v)$, we may assume that $u \geq 0$. 
Now consider the congruences $n 2^{n} \equiv-1(\bmod p)$ and $m_{p} 2^{n_{p}} \equiv-1(\bmod p)$. Observe that $2, n, m_{p}$ are all three coprime to $p$. Raise the first congruence to $u$ and the second to $v$ and multiply them to get

$$
n^{u} m_{p}^{v} 2^{n u+n_{p} v} \equiv(-1)^{u+v} \quad(\bmod p)
$$

Hence, $p$ divides the numerator of the rational number

$$
A:=n^{u} m_{p}^{v} 2^{n u+n_{p} v}-(-1)^{u+v} .
$$

Let us show that $A \neq 0$. Assume that $A=0$. Recall that $n=2{ }^{\alpha} n_{1}$. Thus, expression (41) is

$$
A=n_{1}^{u} m_{p}^{v} 2^{(n+\alpha) u+n_{p} v}-(-1)^{u+v}=0 .
$$

Then $n_{1}^{u} m_{p}^{v}=1,(n+\alpha) u+v n_{p}=0$, and $u+v$ is even. Since $u \geq 0$, it follows that $v \leq 0$. Put $w:=-v$, so $w \geq 0$. There exists an odd positive integer $\rho$ such that $n_{1}=\rho^{w}$ and $m_{p}=\rho^{u}$. Since $u$ and $v$ are coprime and $u+v$ is even, it follows that $u$ and $v$ are both odd. Hence, $w$ is also odd. Also, since $m_{p}$ divides $n_{1}$, it follows that $u \leq w$. We now get

$$
\left(2^{\alpha} \rho^{w}+\alpha\right) u-w n_{p}=0
$$

SO

$$
\frac{u}{n_{p}}=\frac{w}{2^{\alpha} \rho^{w}+\alpha} .
$$

The left-hand side is $\geq u / n=u /\left(2^{\alpha} \rho^{u}\right)$, because $n_{p} \leq n=2^{\alpha} \rho^{u}$. Hence, we get that

$$
\frac{u}{2^{\alpha} \rho^{u}} \leq \frac{u}{n_{p}}=\frac{w}{2^{\alpha} \rho^{w}+\alpha} \quad \text { leading to } \quad \frac{u}{\rho^{u}} \leq \frac{w}{\rho^{w}+\left(\alpha / 2^{\alpha}\right)} \leq \frac{w}{\rho^{w}}
$$

For $\rho \geq 3$, the function $s \mapsto s / \rho^{s}$ is decreasing for $s \geq 0$, so the above inequality together with the fact that $u \leq w$ implies that $u=w$ (so both are 1 because they are coprime), and that all the intermediary inequalities are also equalities. This means that $u=w=1, \alpha=0$ and $n=n_{p}$, but all this is possible only when $C_{n}=p$, which is not allowed. If $\rho=1$, we then get that $n_{1}=1$, so every prime factor $p$ of $C_{n}$ is a Fermat prime. Hence, we get

$$
C_{n}=2^{n_{2}}+1=\prod_{i=1}^{k}\left(2^{2^{\gamma_{p_{i}}}}+1\right)=\sum_{I \subseteq\{1, \ldots, k\}} 2^{\sum_{i \in I} 2^{\gamma_{p_{i}}}},
$$

and $k \geq 2$, but this is impossible by the unicity of the binary expansion of $C_{n}$.

Thus, it is not possible for the expression $A$ shown at (4) to be zero.

The size of the numerator of $A$ is at most

$$
\begin{aligned}
& 2^{1+\left|n u+n_{p} v\right|} n^{u} m_{p}^{|v|} \leq 2^{1+3(n \log n)^{1 / 2}} n^{2(n / \log n)^{1 / 2}} \\
& <2^{1+3(n \log n)^{1 / 2}+(2 / \log 2)(n \log n)^{1 / 2}}<2^{6(n \log n)^{1 / 2}} .
\end{aligned}
$$

In the above chain of inequalities, we used the fact that $3+2 / \log 2<5.9$, together with the fact that $(n \log n)^{1 / 2}>10$ for $n \geq 30$. Thus, for $n \geq 30$, we have that the inequality

$$
p<2^{6(n \log n)^{1 / 2}}
$$

holds for all prime factors $p$ of $C_{n}$. 
Thus, we get the inequality

$$
2^{n}<C_{n}=\prod_{i=1}^{k} p_{i}<\prod_{i=1}^{k} 2^{6(n \log n)^{1 / 2}}=2^{6 k(n \log n)^{1 / 2}},
$$

leading to

$$
k>\frac{n^{1 / 2}}{6(\log n)^{1 / 2}} .
$$

Comparing estimates (3) and (6), we get

$$
\frac{n^{1 / 2}}{6(\log n)^{1 / 2}}<1+2.4 \log n,
$$

implying that $n<6 \times 10^{5}$.

It remains to lower this bound. We first lower it to $n<93000$. Indeed, first note that since $n<6 \times 10^{5}$, it follows that if $p=F_{\gamma}=2^{2^{\gamma}}+1$ is a Fermat prime dividing $C_{n}$, then $\gamma \leq 18$. The only such Fermat primes are for $\gamma \in\{0,1,2,3,4\}$. Furthermore, $(\log n) /(\log 3) \leq \log \left(6 \times 10^{5}\right) /(\log 3)=12.1104 \ldots$ Hence, $k \leq 5+$ $12=17$. It then follows, by equation (6), that

$$
\frac{n^{1 / 2}}{6(\log n)^{1 / 2}}<17
$$

so $n<122000$. But then $(\log n) /(\log 3)<\log (122000) /(\log 3)=10.6605 \ldots$, giving that in fact $k \leq 15$. Inequality (6) shows that

$$
\frac{n^{1 / 2}}{6(\log n)^{1 / 2}}<15,
$$

so $n<93000$. Next let us observe that if $n$ is not a multiple of 3 , then relation (2) leads easily to the conclusion that the number of prime factors $p$ of $C_{n}$ with $m_{p}>1$ is in fact $\leq(\log n) /(\log 5)=7.15338 \ldots$ Hence, the number of such primes is $\leq 7$, giving that $k \leq 12$, which contradicts a result of Cohen and Hagis [5] who showed that every number with the Lehmer property must have at least 14 distinct prime factors. Hence, $3 \mid n$, which shows that $C_{n}$ is not a multiple of 3 . An argument similar to one used before proves that $n$ is not a multiple of any prime $q>3$. Indeed, if it were, then relation (2) would lead to the conclusion that the number of prime factors $p$ of $C_{n}$ with $m_{p}>1$ is $\leq 1+\log (n / q) /(\log 3) \leq 1+\log (93000 / 5) /(\log 3)=$ $9.94849 \ldots$, so there are at most 9 such primes. Also, $C_{n}$ can be divisible with at most 4 of the 5 Fermat primes $F_{\gamma}$ with $\gamma \in\{0,1,2,3,4\}$, because $3=F_{0}$ does not divide $C_{n}$. Hence, $k \leq 9+4=13$, which again contradicts the result from [5]. Thus, $n=2^{\alpha} 3^{\beta}$ and so all prime factors $p$ of $C_{n}$ are of the form $2^{\alpha_{1}} 3^{\beta_{1}}+1$ for some nonnegative integers $\alpha_{1}$ and $\beta_{1}$. Now write

$$
a=\frac{C_{n}-1}{\phi\left(C_{n}\right)}=\prod_{i=1}^{k}\left(1+\frac{1}{p_{i}-1}\right)
$$

for some integer $a \geq 2$. Since

$$
\prod_{\substack{\alpha_{1} \geq 0, \beta_{1} \geq 0 \\ 2^{\alpha_{1}} 3^{\beta_{1}}+1 \text { prime }}}\left(1+\frac{1}{2^{\alpha_{1}} 3^{\beta_{1}}}\right)<1.46
$$


we get that $a<2$, which is a contradiction. This shows that in fact there are no numbers $C_{n}$ with the claimed property.

We end with some challenges for the reader.

Research problem. Prove that $C_{n}$ is not a Carmichael number for any $n \geq 1$.

If this is too hard, can one at least give a sharp upper bound on the counting function of the set $\mathcal{C}$ of positive integers $n$ such that $C_{n}$ is a Carmichael number? We recall that Heppner [7] proved that if $x$ is large, then the number of positive integers $n \leq x$ such that $C_{n}$ is prime is $O(x / \log x)$, whereas in 12 it was shown that if $a>1$ is a fixed integer, then the number of positive integers $n \leq x$ such that $C_{n}$ is base $a$ pseudoprime is $O(x(\log \log x) / \log x)$. Clearly, imposing that $C_{n}$ is Carmichael (which is a stronger condition) should lead to sharper upper bounds for the counting function of such indices $n$.

Finally, here is a problem suggested to us by the referee. Theorem 1 shows that $\phi\left(C_{n}\right) / \operatorname{gcd}\left(C_{n}-1, \phi\left(C_{n}\right)\right)$ exceeds 1 for all $n$. Can one say something more about this ratio? For example, it is possible that a minor modification of the arguments in the paper would show that this function tends to infinity with $n$, but we have not worked out the details of such a deduction. It would be interesting to find a good (large) lower bound on this quantity which is valid for all $n$ and which tends to infinity with $n$. How about for most $n$ ? What about lower and upper bounds on the average value of this function when $n$ ranges in the interval $[1, x]$ and $x$ is a large real number? We leave these questions for further research.

\section{ACKNOWLEDGEMENTS}

We thank the referee for a careful reading of the paper and for suggesting some of the questions mentioned at the end. The second author was supported in part by grants PAPIIT 100508 and SEP-CONACyT 79685.

\section{REFERENCES}

[1] W. D. Banks, A. M. Güloğlu and C. W. Nevans, 'On the congruence $N \equiv A(\bmod \varphi(N))$ ', Integers 8 (2008), \#A59. MR2472077 (2010g:11154)

[2] W. D. Banks and F. Luca, 'Composite integers $n$ for which $\varphi(n) \mid n-1$ ', Acta Math. Sinica 23 (2007), 1915-1918. MR2352307 (2008j:11134)

[3] P. Berrizbeitia and J. G. Fernandes, 'Observaciones sobre la primalidad de los números de Cullen', short communication in "Terceras Jornadas de Teoría de Números" (http://campus.usal.es/ tjtn2009/doc/abstracts.pdf).

[4] J. Cilleruelo and F. Luca, 'Repunit Lehmer numbers', Proc. Edinburgh Math. Soc. 54 (2011), 55-65.

[5] G. L. Cohen and P. Hagis, 'On the number of prime factors of $n$ if $\phi(n) \mid n-1$ ', Nieuw Arch. Wisk. 28 (1980), 177-185. MR582925(81j:10002)

[6] J. M. Grau and A. M. Oller-Marcén, 'An $O\left(\log ^{2} N\right)$ time primality test for generalized Cullen numbers', preprint, 2010, to appear in Math. Comp.

[7] F. Heppner, 'Über Primzahlen der Form $n 2^{n}+1$ bzw. $p 2^{p}+1$ ', Monatsh. Math. 85 (1978), 99-103. MR0567136 (58:27859)

[8] C. Hooley, Applications of sieve methods to the theory of numbers, Cambridge University Press, Cambridge, 1976. MR0404173 (53:7976)

[9] F. Luca, 'Fibonacci numbers with the Lehmer property', Bull. Pol. Acad. Sci. Math. 55 (2007), 7-15. MR2304295 (2008g:11024)

[10] F. Luca, 'On the greatest common divisor of two Cullen numbers', Abh. Math. Sem. Univ. Hamburg 73 (2003), 253-270. MR2028519(2004i:11001)

[11] F. Luca and C. Pomerance, 'On composite integers $n$ for which $\phi(n) \mid n-1$ ', Bol. Soc. Mat. Mexicana, to appear. 
[12] F. Luca and I. E. Shparlinski, 'Pseudoprime Cullen and Woodall numbers', Colloq. Math. 107 (2007), 35-43. MR2283130 (2007i:11127)

[13] C. Pomerance, 'On the distribution of amicable numbers', J. reine angew. Math. 293/294 (1977), 217-222. MR0447087(56:5402)

[14] C. Pomerance, 'On composite $n$ for which $\varphi(n) \mid n-1$, II', Pacific J. Math. 69 (1977), 177-186. MR0434938 (55:7901)

Departamento de Mátematicas, Universidad de Oviedo, Avenida Calvo Sotelo, s/n, 33007 Oviedo, Spain

E-mail address: grau@uniovi.es

Instituto de Matemáticas, Universidad Nacional Autonoma de México, C.P. 58089, Morelia, MichoacÁn, MÉxico

E-mail address: fluca@matmor.unam.mx 